\title{
Methionine and arginine supplementation alter inflammatory and oxidative stress responses during lipopolysaccharide challenge in bovine mammary epithelial cells in vitro
}

\author{
H. Dai, ${ }^{1,2}$ D. N. Coleman, ${ }^{2} \odot$ L. Hu, ${ }^{2,3}$ I. Martinez-Cortés,,${ }^{2,4}$ M. Wang, ${ }^{3}$ C. Parys, ${ }^{5}$ X. Shen, ${ }^{1} \oplus$ and J. J. Loor ${ }^{2 *} \oplus$ \\ ${ }^{1}$ College of Veterinary Medicine, Nanjing Agricultural University, 210095 P. R. China \\ ${ }^{2}$ Department of Animal Sciences and Division of Nutritional Sciences, University of Illinois, Urbana 61801 \\ ${ }^{3}$ College of Animal Science and Technology, Yangzhou University, 225009 P. R. China \\ ${ }^{4}$ Agricultural and Animal Production Department, UAM-Xochimilco, Mexico City 04960 \\ ${ }^{5}$ Evonik Nutrition and Care GmbH, Hanau-Wolfgang, 63457, Germany
}

\section{ABSTRACT}

Mastitis, inflammation of the udder, is one of the most common diseases hampering milk yield of dairy cows. Methionine (Met) and arginine (Arg) are key nutrients with potential to regulate inflammation and oxidative stress. The aim of this study was to evaluate the effect of increased supply of Met and Arg on mRNA and protein abundance associated with innate immune response and redox balance during lipopolysaccharide (LPS) stimulation in primary bovine mammary epithelial cells (BMEC). Primary BMEC ( $\mathrm{n}=4$ replicates per treatment) were pre-incubated for $12 \mathrm{~h}$ in media with the following amino acid combinations: ideal profile of amino acids (control; Con), increased Met supply (incMet), increased Arg supply (incArg), and increased supply of Met and Arg (incMetArg). Subsequently, cells were challenged with or without LPS $(1 \mu \mathrm{g} / \mathrm{mL})$ and incubated for $6 \mathrm{~h}$. Data were analyzed as a $2 \times 2$ $\times 2$ factorial using the MIXED procedure of SAS 9.4 (SAS Institute Inc., Cary, NC). The downregulation of SLC36A1 and SLC7A1 mRNA abundance induced by LPS was attenuated in the incArg cultures. Although challenge with LPS led to lower abundance of proteins related to the antioxidant response (NFE2L2, NQO1, GPX1), lower levels of ATG7, and lower mRNA abundance of GPX3, we found little effect in cultures with incMet or incArg. Cultures with incMet, incArg, or incMetArg led to attenuation of the upregulation of SOD2 and NOS2 induced by LPS. Abundance of phosphorylated p65 (RELA) was greater after LPS stimulation, but the response was attenuated in cultures with

Received March 15, 2019.

Accepted September 24, 2019.

*Corresponding author: jloor@illinois.edu
incMet. The greater ratio of pRELA to total RELA in responses to LPS was also attenuated in cultures with incMetArg. The greater mRNA abundance of the proinflammatory cytokine IL1B induced by LPS was attenuated in cultures with incMet, and the same trend induced by LPS on $C X C L 2$ was also alleviated in cultures with incArg. Overall, the data suggest that greater supply of Met and Arg alleviated the proinflammatory responses triggered by LPS through controlling the abundance of proinflammatory cytokines and chemokines and activity of NF- $\kappa \mathrm{B}$. Little benefit on oxidative stress induced by LPS challenge in BMEC was detected with greater supply of Met and Arg.

Key words: amino acids, immune response, mammary epithelial cell, mastitis

\section{INTRODUCTION}

Mastitis, an inflammation of the mammary gland, is one of the most common diseases in dairy cows. The most common causative pathogens of bovine mastitis are the gram-negative species Escherichia coli and Klebsiella pneumoniae and the gram-positive species Streptococcus agalactiae, Streptococcus uberis, and Staphylococcus aureus (Zadoks et al., 2011). Lipopolysaccharide is a conserved component expressed in the cell walls of a wide range of gram-negative bacteria (Elazar et al., 2010). Innate immune responses initiated by LPS in primary bovine mammary epithelial cells (BMEC) have been widely used in the study of mastitis in vitro (He et al., 2017; Wang et al., 2017b). Toll-like receptors (TLR) are a major class of receptors that recognize pathogen-associated molecular patterns, and TLR4 has been identified as the receptor of LPS (Oliver and Bushe, 1987). Once bound with LPS, TLR4 initiates the activation of nuclear factor $\kappa \mathrm{B}$

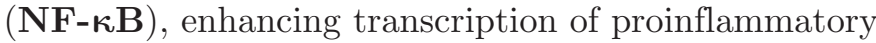
genes (Akira, 2009; Moyes et al., 2010; Li et al., 2015). 
Amino acids are important for immune function and recovery during infectious diseases, but their supply is often diminished during stress or inflammation, especially Met and Arg (Wu, 2013).

Methionine is the first limiting AA for dairy cows. A recent meta-analysis supports a substantial body of research indicating a positive effect of enhanced Met supply for optimal milk protein percentage and yield during lactation (Lean et al., 2018). Beyond production benefits, recent data have revealed a benefit of enhanced Met supply during the periparturient period in terms of alleviating inflammation and oxidative stress (Osorio et al., 2014; Zhou et al., 2016; Batistel et al., 2018). As a sulfur-containing AA, Met is used by cells for the synthesis of the sulfur-containing antioxidants glutathione (GSH) and taurine (Brosnan and Brosnan, 2006; Zhou et al., 2016).

Arginine, a semi-essential AA, plays a crucial role in the maintenance of immune function and exerts antiinflammatory functions (de Andrade Bernal Fagiani et al., 2019). Those characteristics help explain the benefit of Arg in helping reduce postoperative infection (Popovic et al., 2007). Data also highlight the role of Arg as a functional AA (Wu, 2013), with some studies reporting a benefit of enhancing Arg supply in the context of the proinflammatory response triggered by LPS challenge (Wu et al., 2016; Zhao et al., 2018). Beyond these roles, dietary supply of Arg alleviates oxidative stress by enhancing concentrations of GSH (Dasgupta et al., 2006). However, no studies to our knowledge have attempted to elucidate whether increased supplies of Met or Arg to mammary cells (while maintaining an ideal AA profile) during an inflammatory challenge exert a beneficial effect on the biological mechanisms that allow cells to maintain homeostasis.

Primary BMEC are not only important for milk protein production; they are also the first line of defense against the invasion of pathogens. Therefore, our general hypothesis was that enhanced Met or Arg supply based on an ideal EAA profile (Haque et al., 2012, 2013) modulates the proinflammatory and antioxidant response of mammary cells during inflammation to mimic mastitis. The same profiles of AA have previously been used in cell culture studies from our laboratory (Li et al., 2016; Dong et al., 2018; Vailati-Riboni et al., 2019). The aim of the present study was to evaluate whether supplementation of Met or Arg, alone or in combination, in the medium of isolated primary BMEC, modulates immune and antioxidant responses triggered by LPS in vitro. The mRNA and protein abundance data for a wide number of targets were measured to better capture potential mechanistic roles for these AA during inflammation.

\section{MATERIALS AND METHODS}

\section{Cell Culture and Treatment}

The BMEC used in the present study were obtained from Yangzhou University (China). Methods used for cell isolation and purification were described previously by Wang et al. (2014). Briefly, mammary tissue obtained from 3 lactating dairy cows free of clinical disease was digested using Type II collagenase in a constant-temperature shaker at $37^{\circ} \mathrm{C}$ for $1 \mathrm{~h}$, and then run through an 80-mesh filter to separate cells from tissue debris. Cells were collected via centrifugation and cultured in $37^{\circ} \mathrm{C}$. To purify epithelial cells from fibroblasts, $0.25 \%$ trypsin was used. The purified epithelial cells were assessed for purity via morphology and immunofluorescence (Wang et al., 2014).

A total of $2 \times 10^{5}$ cells were seeded into 6 -well plates and incubated overnight with serum-free high-glucose Dulbecco's modified Eagle's medium. Cells were then cultured for $12 \mathrm{~h}$ with modified medium: medium with ideal AA profile (control; Con), medium with increased Met supply (incMet), medium with increased Arg supply (incArg) or medium with increased Met and Arg supply (incMetArg). After 12-h incubation in modified medium, cells were treated with either 1 $\mu \mathrm{g} / \mathrm{mL}$ of LPS from E. coli O111:B4 (Sigma-Aldrich, St. Louis, MO) or PBS, followed by another 6 h-incubation. Therefore, there were a total of 8 treatments: (1) Con, (2) incMet, (3) incArg, (4) incMetArg, (5) Con+LPS, (6) incMet+LPS, (7) incArg+LPS, and (8) incMetArg+LPS.

The modified medium used for treatments was serumfree lactogenic, prepared with customized high-glucose Dulbecco's modified Eagle's medium according to Dong et al. (2018). The formulation of the essential AA was as follows: control medium with the ideal AA ratio (Con; Lys:Met 2.9:1; Lys: Arg 2:1; Thr:Phe 1.05:1; Lys:Thr 1.8:1; Lys:His 2.38:1; Lys:Val 1.23:1), incMet (Lys:Met 2.5:1), incArg (Lys:Arg 1:1), and incMetArg (Lys:Met 2.5:1; Lys:Arg 1:1). Media were prepared by increasing only Met, only Arg, or both, while keeping the other AA ratios the same as in Con (described in Table 1). At the end of this incubation, cells were collected using either Qiazol regent (Qiagen, Valencia, CA) or Mammalian Protein Extraction Reagent (M-PER; Thermo Fisher Scientific, Waltham, MA), for mRNA and protein analyses. The media used were purchased from Gibco (Invitrogen, Carlsbad, CA), and L-isomers of the individual EAA were purchased from Sigma-Aldrich. All treatments were performed with 4 replicates, and incubations were conducted in a humidified incubator at $37^{\circ} \mathrm{C}$ and an atmosphere of $5 \% \mathrm{CO}_{2}$. 


\section{RNA Extraction, cDNA Synthesis, and Real-Time PCR}

Total RNA from the BMEC was isolated with miRNeasy Mini Kit (Qiagen) according to the manufacturer's instruction (Supplemental File S1; https://doi .org/10.3168/jds.2019-16631). Concentration of total RNA was measured with a ND-1000 spectrophotometer (NanoDrop Technologies, Wilmington, DE), and the RNA integrity number was determined with the Agilent 2100 bioanalyzer (Agilent Technologies, Santa Clara, CA). All samples used for analysis had an integrity number $>8.0$. Synthesis of cDNA and $\mathrm{qPCR}$ were performed according to Dong et al. (2018). In brief, $100 \mathrm{ng}$ of total RNA was mixed with $1 \mu \mathrm{L}$ of Random Primers (Cat. \#11034731001, Roche, Basel, Switzerland) and $9 \mu \mathrm{L}$ of nuclease-free water and incubated at $65^{\circ} \mathrm{C}$ for $5 \mathrm{~min}$. A total of $9 \mu \mathrm{L}$ of master mix was added, composed of $4 \mu \mathrm{L}$ of $5 \times$ First-Strand Buffer (Cat. \#18064-022, Invitrogen), $1 \mu \mathrm{L}$ of Oligo dT18 (Operon Biotechnologies, Huntsville, AL), $2 \mu \mathrm{L}$ of $10 \mathrm{~m} M$ dNTP mix (Cat. \#18427-088, Invitrogen), $1.625 \mu \mathrm{L}$ of nuclease-free water, $0.25 \mu \mathrm{L}(200 \mathrm{U} / \mu \mathrm{L})$ of Revert Aid Reverse Transcriptase (Cat. \#EP0442, Thermo Fisher Scientific), and $0.125 \mu \mathrm{L}(40 \mathrm{U} / \mu \mathrm{L})$ of RiboLock RNase Inhibitor (Cat. \#EO0382, Thermo Fisher Scientific), and the mixture was incubated in an Eppendorf Mastercycler Gradient (Hamburg, Germany), with temperature program as follows: $25^{\circ} \mathrm{C}$ for $5 \mathrm{~min}, 50^{\circ} \mathrm{C}$ for $60 \mathrm{~min}$, and $70^{\circ} \mathrm{C}$ for $15 \mathrm{~min}$.

The cDNA was then diluted 1:4 with nuclease-free water and $4 \mu \mathrm{L}$ of diluted cDNA combined with $6 \mu \mathrm{L}$ of mixture composed of $5 \mu \mathrm{L} 1 \times$ SYBR Green master mix (Cat. \#4309155, Applied Biosystems, Waltham, $\mathrm{MA}), 0.4 \mu \mathrm{L}$ each of $10 \mu M$ forward and reverse primers and $0.2 \mu \mathrm{L}$ of nuclease-free water were added in a MicroAmp Optical 384-Well Reaction Plate (Cat. \#4309849, Applied Biosystems). Reactions were conducted in an ABI Prism 7900 HT SDS instrument (Applied Biosystems) with the following conditions: 2 min at $50^{\circ} \mathrm{C}, 10 \mathrm{~min}$ at $95^{\circ} \mathrm{C}, 40$ cycles of $15 \mathrm{~s}$ at $95^{\circ} \mathrm{C}$, and $1 \mathrm{~min}$ at $60^{\circ} \mathrm{C}$. The threshold cycle data were analyzed and transformed using the standard curve with the 7900 HT Sequence Detection Systems Software (version 2.2.1, Applied Biosystems). The final data were normalized with the geometric mean of the 3 internal controls (Dong et al., 2018). Genes related to amino acid transport, immune response, antioxidant response, and Met and Arg metabolism were assessed, and all reactions were run in duplicate. Information on the primers used in the experiments is listed in Table 2. Detailed information on the design and testing of target primers and $\mathrm{qPCR}$ performance are provided in Supplemental File S1 (https://doi.org/10.3168/jds.2019-16631).

Table 1. Amino acid composition of the ideal AA mixture control (Con) and the treatments containing increased levels of Met, Arg, or both (incMet, incArg, and incMetArg); bovine mammary epithelial cells were preincubated with modified medium containing AA mixtures as described below for $12 \mathrm{~h}$, followed by 6-h LPS stimulation

\begin{tabular}{lcccc}
\hline Amino acid & Con $^{1,2}$ & incMet $^{3}$ & incArg $^{4}$ & incMetArg \\
\hline L-lysine $(\mu \mathrm{g} / \mathrm{mL})$ & 175 & 175 & 175 & 175 \\
Lys:Met & $2.9: 1$ & $2.5: 1$ & $2.9: 1$ & $2.5: 1$ \\
Lys:Arg & $2: 1$ & $2: 1$ & $1: 1$ & $1: 1$ \\
L-methionine $(\mu \mathrm{g} / \mathrm{mL})$ & 60 & 70 & 60 & 70 \\
L-arginine $(\mu \mathrm{g} / \mathrm{mL})$ & 84 & 84 & 175 & 775 \\
L-histidine $(\mu \mathrm{g} / \mathrm{mL})$ & 74 & 74 & 74 & 74 \\
L-isoleucine $(\mu \mathrm{g} / \mathrm{mL})$ & 121 & 121 & 121 & 206 \\
L-leucine $(\mu \mathrm{g} / \mathrm{mL})$ & 206 & 206 & 206 & 93 \\
L-phenylalanine $(\mu \mathrm{g} / \mathrm{mL})$ & 93 & 93 & 93 & 97 \\
L-threonine $(\mu \mathrm{g} / \mathrm{mL})$ & 97 & 97 & 97 & 16 \\
L-tryptophan $(\mu \mathrm{g} / \mathrm{mL})$ & 16 & 16 & 16 & 142 \\
L-valine $(\mu \mathrm{g} / \mathrm{mL})$ & 142 & 142 & 142 &
\end{tabular}

${ }^{1}$ Con $=$ ideal AA profile, used as control medium. Ratio of EAA as follows: Lys:Met $=2.9$, Lys: Thr $=1.8$, Lys: His $=2.38$, Lys:Val $=1.23$, and Thr:Phe $=1.05$, as described in NRC (2001), Haque et al. (2012), and our previous studies: Li et al. (2016) and Vailati-Riboni et al. (2019).

${ }^{2}$ Composition of AA in the medium was prepared as described by Dong et al. (2018).

${ }^{3}$ Increased level of Met was selected based on our previous study (Dong et al., 2018), in which alteration of cellular metabolites was observed when increased Met was supplied (Lys:Met $=2.5: 1$ ).

${ }^{4}$ Increased level of Arg was selected according our previous work (Salama et al., 2019), where positive effect of increased Arg supply (Lys:Arg = 1:1) was observed during heat stress. 
Dai et al.: AMINO ACIDS AND MAMMARY CELL INFLAMMATION

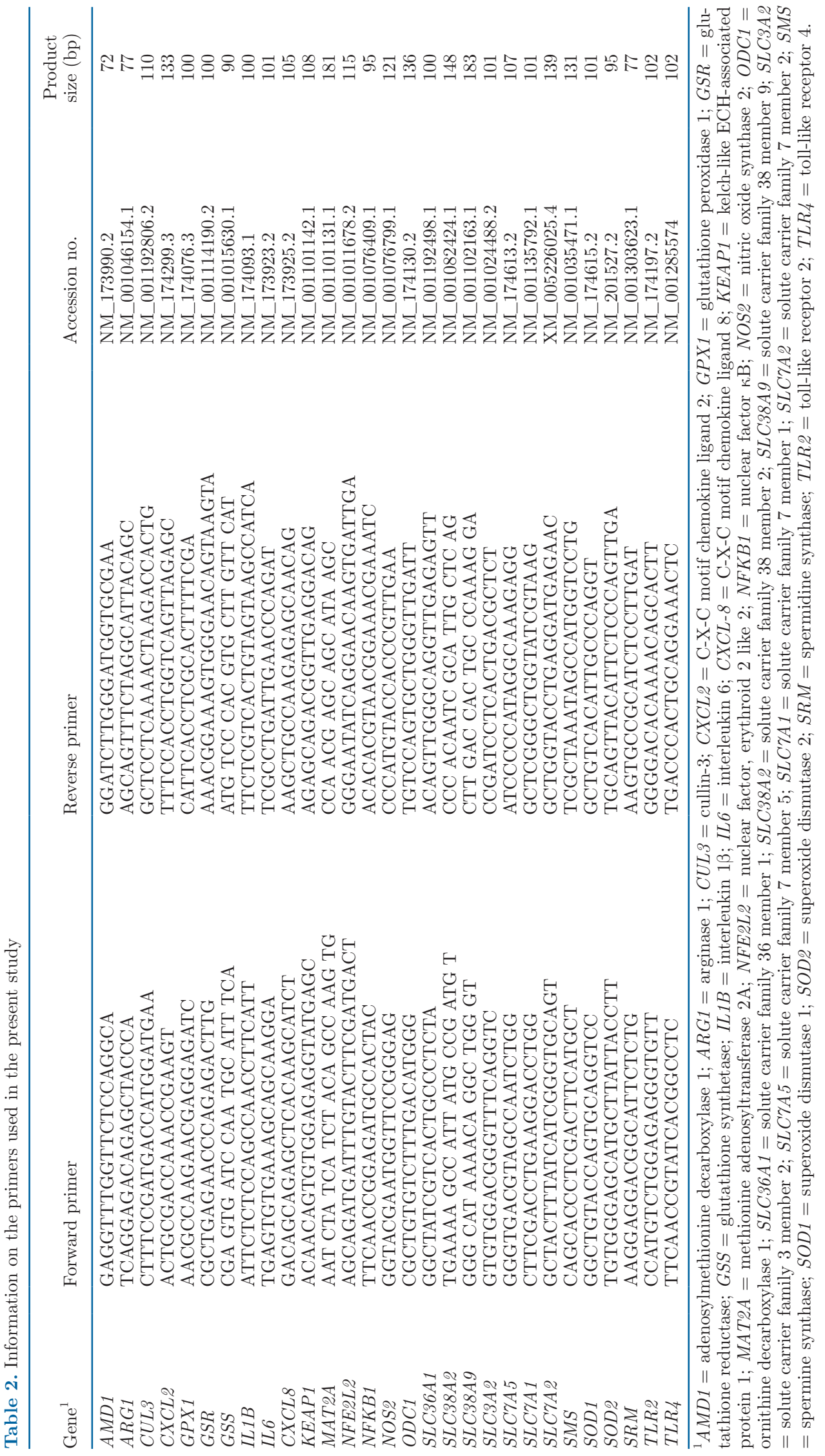




\section{Western Blot Analysis}

The protocol for Western blot analysis was performed as described by Hosseini et al. (2015), and a detailed description is presented in Supplemental File S1 (https://doi.org/10.3168/jds.2019-16631). Briefly, $30 \mu \mathrm{g}$ of total protein of each sample was separated on 4 to $20 \%$ Mini-PROTEAN TGX Precast Protein Gels (\#4561096, Bio-Rad, Hercules, CA) and transferred onto a polyvinylidene difluoride (PVDF) membrane. The membrane was then subjected to a 2 -h blocking in 5\% skim milk or BSA at room temperature. Subsequently, the membrane was incubated with the primary antibodies [anti-CUL3, anti-E1-ubuquitin activating enzyme, anti-GPX3, anti-HMOX1, antiNQO1, anti-Nrf2, anti-NF- $\kappa$ B p65(RELA) (C22B4), anti-phospho-Nrf2 (Ser40), anti-phospho-NF- $\kappa$ B p65 (RELA) (Ser468), anti-GAPDH] overnight at $4^{\circ} \mathrm{C}$. After 65 -min washings with $1 \times$ Tris-buffered saline-Tween buffer, the membrane was incubated with the secondary antibody at room temperature for $1 \mathrm{~h}$, followed by the same wash step. The PVDF membranes were visualized with the Clarity Western ECL substrate (no. 170-5060; Bio-Rad) and scanned using the ChemiDoc MP system (Bio-Rad). Band intensities were quantified using Image Lab software (version 3.0, Bio-Rad), and glyceraldehyde-3-phosphate dehydrogenase was used as the internal reference protein to normalize protein intensity. More detailed information of the antibodies used in present study is reported in Supplemental File S2 (https://doi.org/10.3168/jds.2019-16631).

\section{Statistical Analysis}

Data were analyzed as a $2 \times 2 \times 2$ factorial arrangement of treatments using the MIXED procedure of SAS 9.4 (SAS Institute Inc., Cary, NC). The 3 factors were Met, Arg, and LPS, each containing 2 levels: basal or increased levels of the AA and with or without LPS, resulting in 8 treatments. The model included the main effects of Met, Arg, and LPS, as well as all the following interactions: Met $\times \operatorname{Arg} \times$ LPS, Met $\times$ LPS, $\operatorname{Arg} \times$ LPS, and Met $\times$ Arg. The random effect was individual cell culture well. Variables were assessed for normality of distribution using the Shapiro-Wilk test. Data on mRNA abundance were $\log _{2}$-scale transformed to fit normal distribution of residuals. The tables and graphs contain the $\log _{2}$ back-transformed means that resulted from the statistical analysis. Least squares means and standard errors were determined using the LSMEANS statement of SAS (SAS Institute Inc.) and were compared using Tukey's test when significant interactions were observed. Significance was set at $P \leq 0.05$ and tendencies at $P \leq 0.10$.

\section{RESULTS}

\section{mRNA Abundance}

Amino Acid Transporters. Only 1 significant 3 -way interaction was observed for solute carrier (SLC) family 36 member 1 (SLC36A1; $P=0.01$, Table 3 ), with abundance being lower with LPS relative to Con $(P=0.01)$. However, abundance was greater with incArg + LPS $(P=0.003)$ compared with Con+LPS. A 2-way interaction between Arg and LPS was observed for SLC7A1 $(P<0.001$, Table 3); abundance was lower with LPS compared with cultures without LPS $(P<$ 0.01), whereas cultures with incArg+LPS attenuated the downregulation of $S L C 7 A 1$ abundance in response to LPS stimulation $(P=0.14)$. An interaction between Arg and LPS was also observed for SLC7A5 $(P<$ 0.001, Table 3), where LPS stimulation downregulated $S L C^{\prime} 7 A 5$ relative to the group with no LPS and no increase in $\operatorname{Arg}$ supply $(P<0.001)$. However, culture with incArg+LPS did not rescue the downregulation of $S L C 7 A 5$ abundance induced by LPS $(P=0.82)$. Culture with incArg, incMet, or incArgMet did not attenuate the downregulation of SLC3A2 and SLC7A2 abundance $(P>0.05$, Table 3$)$ induced by LPS stimulation.

NFE2L2 Signaling. A triple interaction between Met, Arg, and LPS was observed for glutathione reductase (GSR;P $=0.04$, Figure 1) and glutathione peroxidase (GPX) 1 (GPX1; $P=0.04$, Figure 1), along with a tendency for a triple interaction for superoxide dismutase (SOD) $2(S O D 2 ; P=0.10$, Figure 1). Lower mRNA abundance of $G S R$ was observed in response to LPS despite being nonsignificant compared with Con $(P=0.17)$; however, compared with cultures with Con+LPS, GSR was upregulated in cultures with incArg+LPS $(P=0.02)$. Abundance of $G P X 1$ was lower in cultures with incMet+LPS $(P=0.01)$ and incArg+LPS $(P=0.01)$ compared with Con+LPS. An upregulation of SOD2 mRNA was observed in response to LPS stimulation compared with control, but its abundance was lower for incArg+LPS $(P=0.03)$ and incMet + LPS $(P=0.06)$ relative to Con + LPS.

We found a 2-way interaction between Arg and LPS $(P=0.03$, Table 3$)$ for the mRNA abundance of the nuclear factor, erythroid 2 like 2 (NFE2L2) regulator cullin-3 (CUL3), where downregulation of mRNA abundance of this gene $(P=0.05)$ was ameliorated in cultures with incArg $(P=0.04$, Table 3$)$. A tendency for a Met $\times$ LPS interaction was observed for kelch-like ECH-associated protein 1 (KEAP1; $P=0.09$, Table 3 ), where treatment with LPS upregulated abundance compared with cultures without LPS $(P=0.02)$, whereas KEAP1 was further upregulated in cultures 
Dai et al.: AMINO ACIDS AND MAMMARY CELL INFLAMMATION

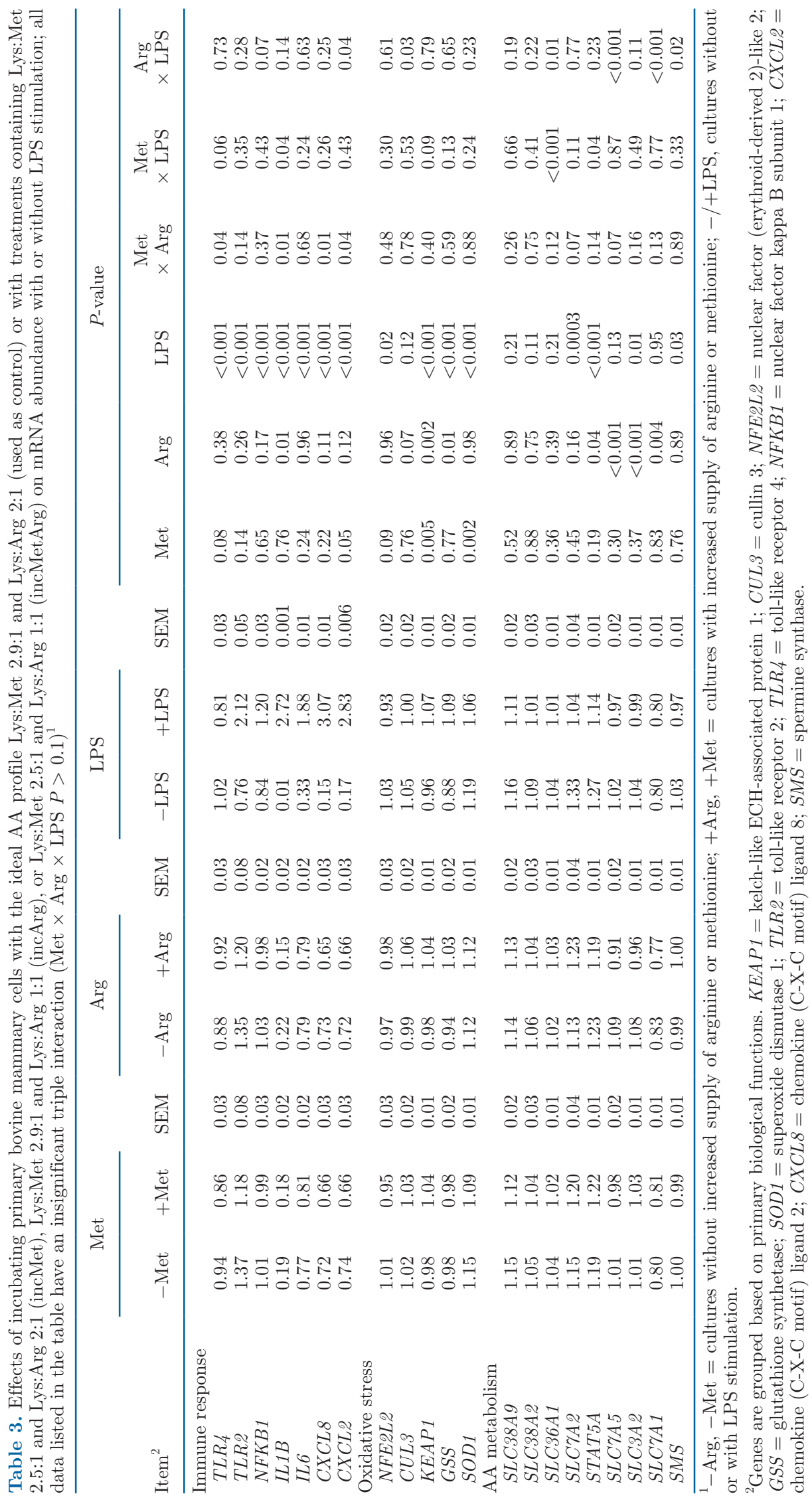




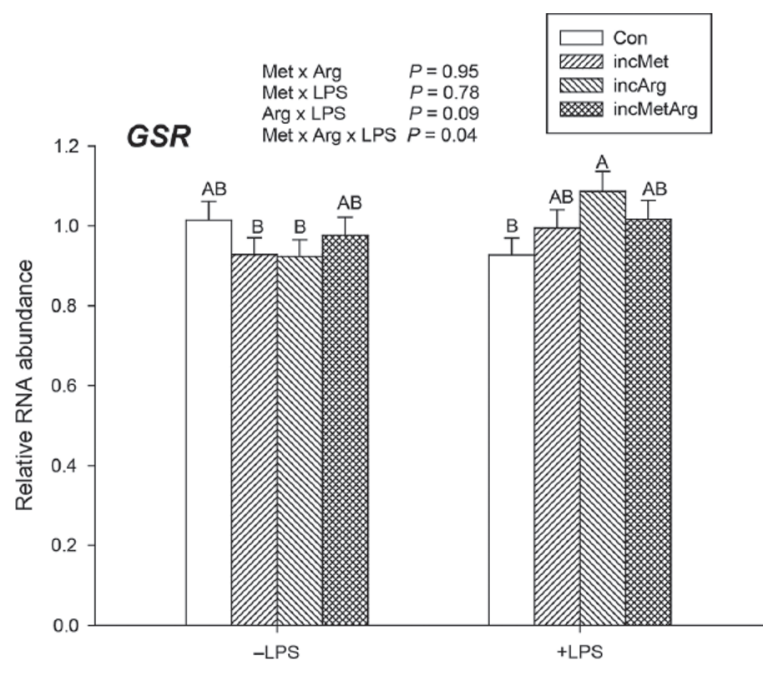

with incMet+LPS $(P=0.01)$. A significant effect of LPS was observed for NFE2L2 $(P=0.02$, Table 3$)$, SOD1 $(P<0.001$, Table 3$)$, and glutathione synthase (GSS; $P<0.001$, Table 3), where their abundance decreased when cells were treated with LPS. Culture with incArg, incMet, or incArgMet did not rescue the downregulation of NFE2L2 and SOD1 induced by LPS.

Immune Response. We discovered a tendency for a 2-way interaction between Met and LPS for TLR4 $(P=0.07)$; stimulation with LPS decreased abundance compared with cells in the cultures without LPS $(P=$ 0.04). Additionally, culture with incMet led to lower abundance of TLR 4 compared with cells cultured with LPS only $(P=0.01$; Table 3$)$. A 2 -way interaction between Met and LPS was observed for $\operatorname{IL1B}(P=0.04)$. Compared with cultures without LPS $(P<0.001)$, the mRNA abundance of $I L 1 B$ was greater with LPS. However, compared with LPS alone, abundance tended to be lower when cells were cultured with incMet+LPS $(P=0.08)$.

We observed an Arg $\times$ LPS interaction for C-X-C motif chemokine ligand $2(\boldsymbol{C X C L 2}, P=0.04)$ due to its abundance being upregulated by LPS stimulation compared with cultures without LPS $(P<0.001$, Table $3)$. Culture with incArg+LPS tended to attenuate the upregulation of $C X C L 2$ abundance induced by LPS stimulation $(P=0.06)$. There was a tendency for an Arg $\times$ LPS interaction for the abundance of the transcription regulator $\operatorname{NFKB1}(P=0.07$, Table 3$)$, with the pattern of change in $N F K B 1$ being similar to that of $C X C L$ 2.

Arginine Metabolism. Triple interactions between Met, Arg, and LPS were observed for spermidine synthase $(S R M ; P=0.01$, Figure 2$)$ and arginase 1 ( $A R G 1$ : $P=0.02$, Figure 2), whereas tendency occurred for a triple interaction for ornithine decarboxylase 1 ( $O D C \mathbf{C}$; $P=0.06$, Figure 2$)$ and nitric oxide (NO) synthase 2 (NOS2; $P=0.06$, Figure 2). Compared with Con $(P$ $=0.03)$ and incArg + LPS cultures $(P=0.005), S R M$ abundance was lower with Con+LPS. The abundance of $A R G 1$ was greater in cultures with incArg+LPS $(P$ $=0.07$, Figure 2$)$ and incMet+LPS $(P=0.08$, Figure 2$)$ compared with Con cultures. Abundance of $O D C 1$ with incArg+LPS was upregulated compared with Con culture $(P=0.02)$ and culture with Con + LPS $(P=0.02)$. Compared with Con cultures, greater mRNA abundance of NOS2 was observed in cells stimulated with LPS $(P<0.001)$, but in cultures with incMet+LPS $(P$ $=0.03)$, incArg + LPS $(P=0.02)$, and incMetArg + LPS $(P=0.03)$, the abundance of NOS2 was lower relative to cultures with Con+LPS. An Arg $\times$ LPS interaction was observed for spermine synthase $(S M S ; P=0.02$, Table 3), with abundance being lower when cells were treated with LPS compared with cultures without LPS

Figure 1. Effects of incubating primary bovine mammary cells with the ideal AA profile Lys:Met 2.9:1 and Lys:Arg 2:1 (Con) or with Lys:Met 2.5:1 and Lys:Arg 2:1 (incMet), Lys:Met 2.9:1 and Lys: Arg 1:1 (incArg), or Lys:Met 2.5:1 and Lys:Arg 1:1 (incMetArg) on mRNA abundance of antioxidant genes, with (+) or without (-) LPS stimulation. Different uppercase letters indicate significant differences between treatments $(P<0.05)$ with a significant triple interaction $($ Met $\times \operatorname{Arg} \times$ LPS $P \leq 0.10)$. Error bars represent SEM. GSR $=$ glutathione-disulfide reductase; $S O D 2=$ superoxide dismutase $2 ; G P X 1$ $=$ glutathione peroxidase 1 . 
$(P=0.003$, Table 3$)$. However, compared with LPS alone, the cultures with incArg or incArg+LPS tended to have greater abundance of $S M S(P<0.10$, Table 3$)$.
Methionine Metabolism. A Met $\times$ Arg $\times$ LPS interaction was observed for both adenosylmethionine decarboxylase 1 ( $\boldsymbol{A} \boldsymbol{M D} \mathbf{1} ; P=0.04$, Figure 2$)$ and me-
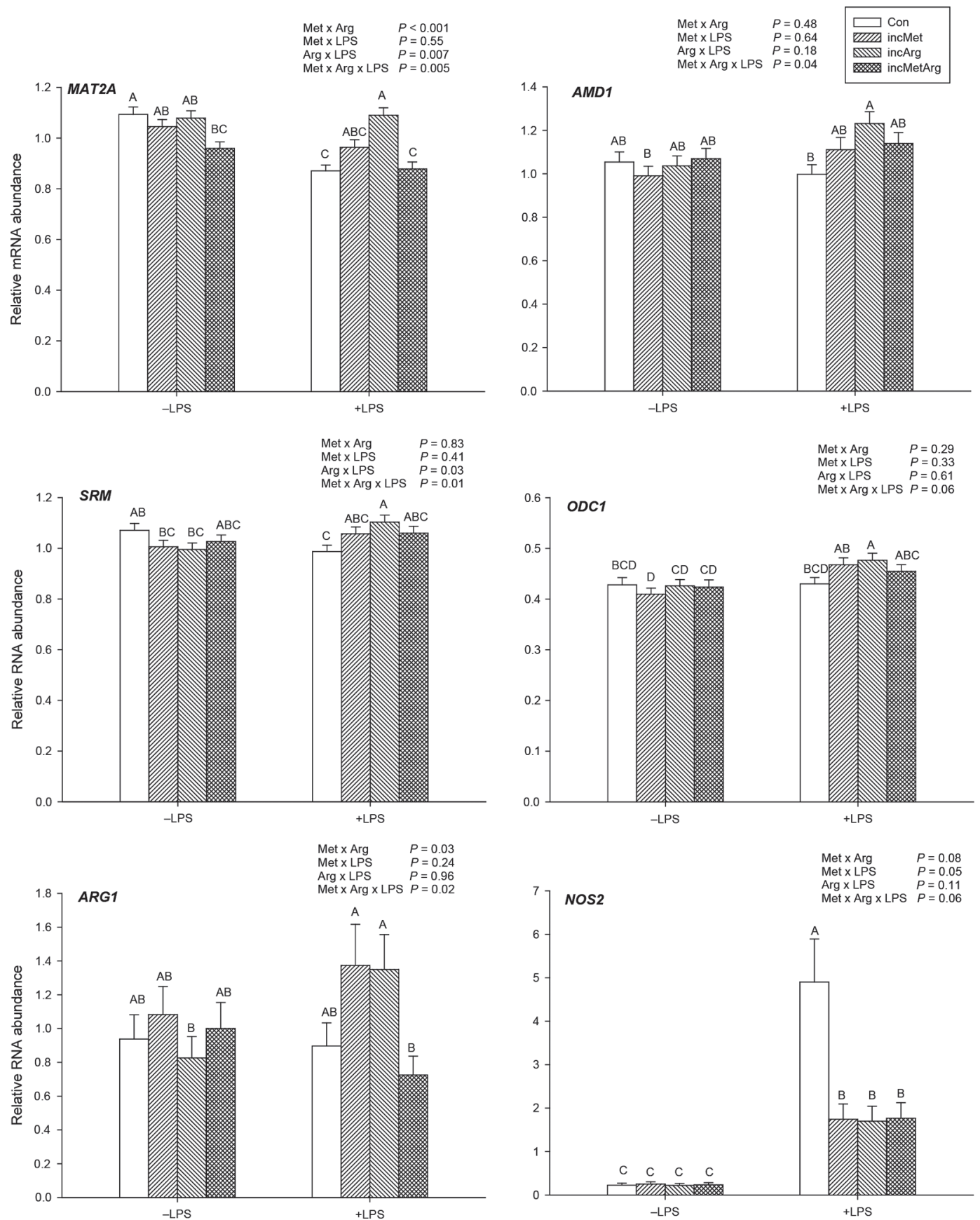

Figure 2. Effects of incubating primary bovine mammary cells with the ideal AA profile Lys:Met 2.9:1 and Lys:Arg 2:1 (Con) or with Lys: Met 2.5:1 and Lys:Arg 2:1 (incMet), Lys:Met 2.9:1 and Lys:Arg 1:1 (incArg), or Lys:Met 2.5:1 and Lys:Arg 1:1 (incMetArg) on mRNA abundance of the genes involved in Arg and Met metabolism, with (+) or without (-) LPS stimulation. Different uppercase letters indicate significant difference between treatments $(P \leq 0.05)$ with a significant triple interaction (Met $\times \operatorname{Arg} \times \operatorname{LPS} P<0.06)$. Error bars represent SEM. SRM $=$ spermidine synthase; $O D C 1=$ ornithine decarboxylase $1 ; N O S 2=$ nitric oxide synthase 2 , inducible; $A M D 1=$ adenosylmethionine decarboxylase 1; MAT2A = methionine adenosyl-transferase 2A. 
thionine adenosyltransferase 2A $(M A T 2 A ; P=0.01$, Figure 2). Abundance of $A M D 1$ was greater in cultures with incArg+LPS $(P=0.04)$ compared with Con+LPS culture. Abundance of MAT2A mRNA in cultures with Con + LPS $(P<0.01)$ and incMetArg + LPS $(P<0.01)$ was greater compared with Con culture, and greater abundance of MAT2A in cultures with incArg+LPS ( $P$ $<0.01)$ was observed relative to culture with Con+LPS and incMetArg+LPS $(P<0.01)$.

\section{Protein Abundance}

A triple interaction between Met, Arg, and LPS was observed for protein abundance of p65 (RELA) $(P=$ 0.04 , Figure 3$)$. An overall greater protein abundance of RELA was detected for cultures with incArg+LPS compared with incArg culture $(P=0.03)$. Additionally, compared with Con+LPS $(P=0.03)$, incMet+LPS $(P=0.04)$, and incArg + LPS $(P=0.01)$ culture, incMetArg+LPS had lower RELA protein abundance. A Met $\times$ LPS interaction occurred for the protein abundance of phosphorylated p65 (pRELA; $P<0.001$, Table 4), abundance of pRELA being greater when cells were stimulated with LPS only compared with cells treated with incMet+LPS $(P<0.001$, Table 4$)$ or without LPS and increased Met supply $(P<0.001$, Table 4). A tendency also existed for a triple interaction of Met, Arg, and LPS for the ratio of pRELA to total RELA ( $P=0.10$, Figure 3$)$; except for culture with incMetArg+LPS $(P=0.14)$, stimulation with LPS increased the ratio compared with all the other treatments $(P<0.05$, Figure 3$)$, whereas the ratio was lower in incMet+LPS culture compared with Con+LPS culture $(P<0.05$, Figure 3$)$

A significant Met $\times \operatorname{Arg} \times$ LPS interaction occurred for the protein abundance of heme oxygenase 1 (HMOX1, $P=0.001$, Figure 4). Abundance of HMOX1 was lower in cells stimulated with LPS $(P<0.01)$ relative to control cells, whereas it was greater in culture with incArg+LPS $(P<0.01)$ and incMetArg+LPS $(P$ $=0.003)$ compared with Con+LPS. Protein abundance of CUL3 was greater in response to LPS stimulation relative to cells without LPS $(P<0.001$, Table 4$)$, but culture with incArg, incMet, and incMetArg did not affect the upregulation of CUL3 abundance induced by LPS. We found a tendency for an Arg $\times$ LPS interaction $(P=0.08)$ for NFE2L2 protein abundance $(P=$ 0.08 , Table 4); LPS stimulation decreased abundance compared with culture with incArg $(P<0.001)$ or cultures without LPS stimulation $(P<0.001)$ but was not different compared with incArg+LPS culture $(P=$ $0.44)$.
We discovered a tendency for a Met $\times$ Arg $\times$ LPS interaction for the ratio of phosphorylated NFE2L2 (pNFE2L2) to total NFE2L2 protein $(P=0.10$, Figure 4) and a significant Met $\times \operatorname{Arg} \times$ LPS interaction for GPX3 $(P=0.001$, Figure 4$)$ and $\mathrm{NAD}(\mathrm{P}) \mathrm{H}$ [quinone] dehydrogenase (NQO1; $P<0.001$, Figure 4 ). The ratio of pNFE2L2 to total NFE2L2 protein was greater
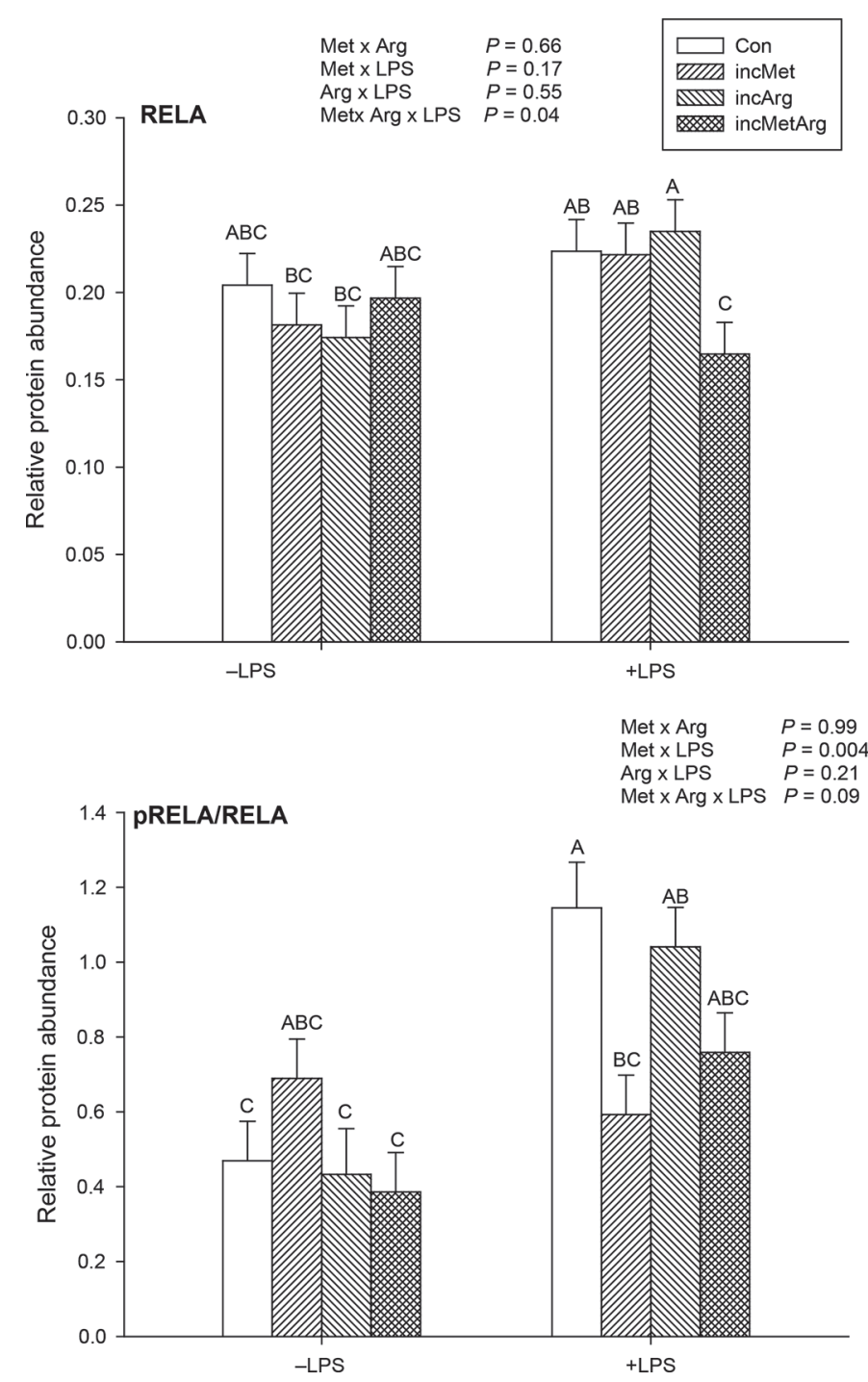

Figure 3. Effects of incubating primary bovine mammary cells with the ideal AA profile Lys:Met 2.9:1 and Lys:Arg 2:1 (Con) or with Lys:Met 2.5:1 and Lys:Arg 2:1 (incMet), Lys:Met 2.9:1 and Lys:Arg 1:1 (incArg), or Lys:Met 2.5:1 and Lys:Arg 1:1 (incMetArg) on the NF- $\kappa \mathrm{B}$ p65 (RELA) protein abundance in response to LPS challenge $(+$ LPS $=$ LPS challenge; - LPS $=$ no LPS stimulation $)$. All data are reported as relative protein abundance of the target versus GAPDH, which served as the internal control. Different uppercase letters indicate significant differences between treatments $(P<0.05)$ with a significant triple interaction (Met $\times \operatorname{Arg} \times$ LPS $P<0.05)$. Error bars denote SEM. 


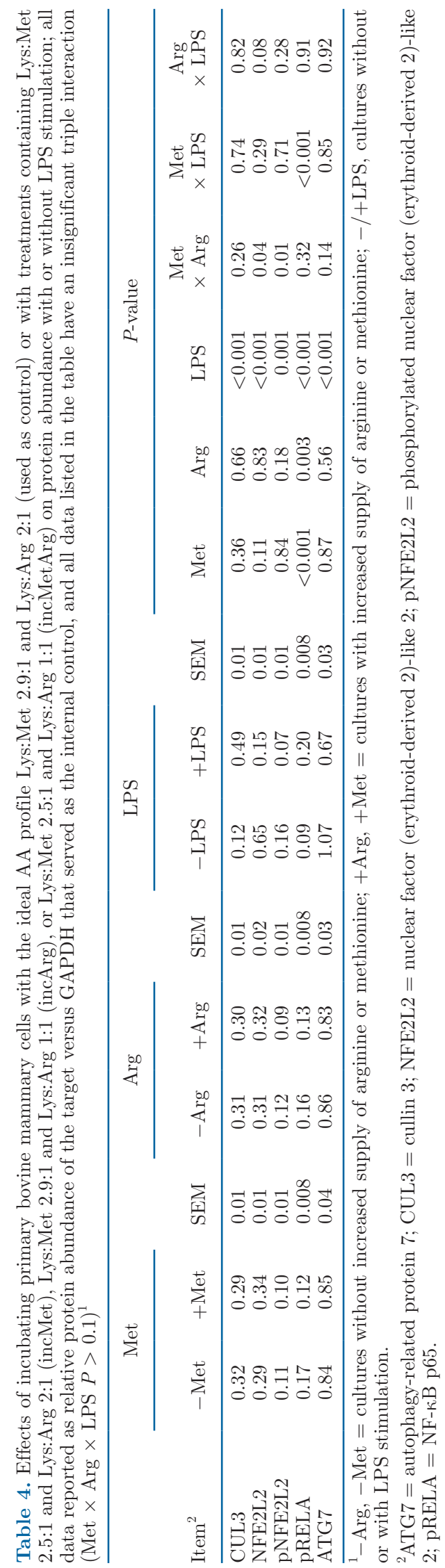

in Con+LPS cultures compared with Con cultures $(P$ $<0.01$, Figure 4$)$, but it was lower in cultures with incMet+LPS $(P=0.03)$ and incArg+LPS $(P<0.01)$ relative to Con + LPS. Stimulation with LPS decreased the protein abundance of GPX3 $(P<0.001$, Figure 4$)$, NQO1 $(P<0.001$, Figure 4$)$, and autophagy-related protein 7 (ATG ${ }^{r} ; P<0.001$, Table 4$)$; however, culture with incArg, incMet, and incMetArg did not attenuate the downregulation of these proteins induced by LPS.

\section{DISCUSSION}

Amino acid transporters play crucial roles in delivery of AA to cells and also participate in key intracellular signaling pathways (Bröer and Palacin, 2011). The upregulation of $S L C 36 A 1$ and $S L C 7 A 1$ mRNA abundance with increased Arg supply during LPS stimulation suggests that Arg attenuated the decrease in SLC36A1 and SLC7A1 expression resulting from the LPS challenge. Upon initiation of an inflammatory response via LPS, a concomitant decrease in plasma concentrations of Arg has been observed (Bansal and Ochoa, 2003), suggesting a potential benefit for increasing the supply of Arg. The gene SLC7A1 encodes a transporter for Arg and other cationic AA (Bronte and Zanovello, 2005) and is upregulated in LPS-stimulated macrophages (Closs et al., 2000). Thus, increased abundance of SLC7A1 with Arg supplementation during an LPS challenge may represent a mechanism for mammary cells to ensure sufficient supply of Arg, as sufficient Arg is crucial for synthesis of NO, which exerts a bactericidal property during inflammation ( $\mathrm{Li}$ et al., 2007). The gene SCL36A1 encodes a transporter of Pro, Ala, and other AA. The upregulation of SLC36A1 in cultures with incArg+LPS compared with Con+LPS cultures in the present study is comparable to previous work, which found that LPS stimulation caused a downregulation in SLC36A1 and that the supply of Arg reversed the effect (Jensen et al., 2014). Upregulation of this transporter would facilitate the uptake of AA, which may help attenuate or modulate immune responses by BMEC.

Adenosylmethionine decarboxylase 1 (AMD1) is a key enzyme in Met metabolism, converting S-adenosyl methionine to S-adenosylmethionine amine (Pegg et al., 1998). This is essential for the synthesis of polyamines (Pegg et al., 1998), which can elicit an antiinflammatory response (Zhang et al., 1997). Thus, the upregulation of $A M D 1$ (a crucial intermediate enzyme in polyamine biosynthesis) in the present study, in cultures with incArg during LPS stimulation, supports the idea of polyamine generation as an important component of the anti-inflammatory response induced by Arg.

Along with the upregulation of AMD1, the upregulation of $O D C 1$ and $S M S$ abundance in cultures with 

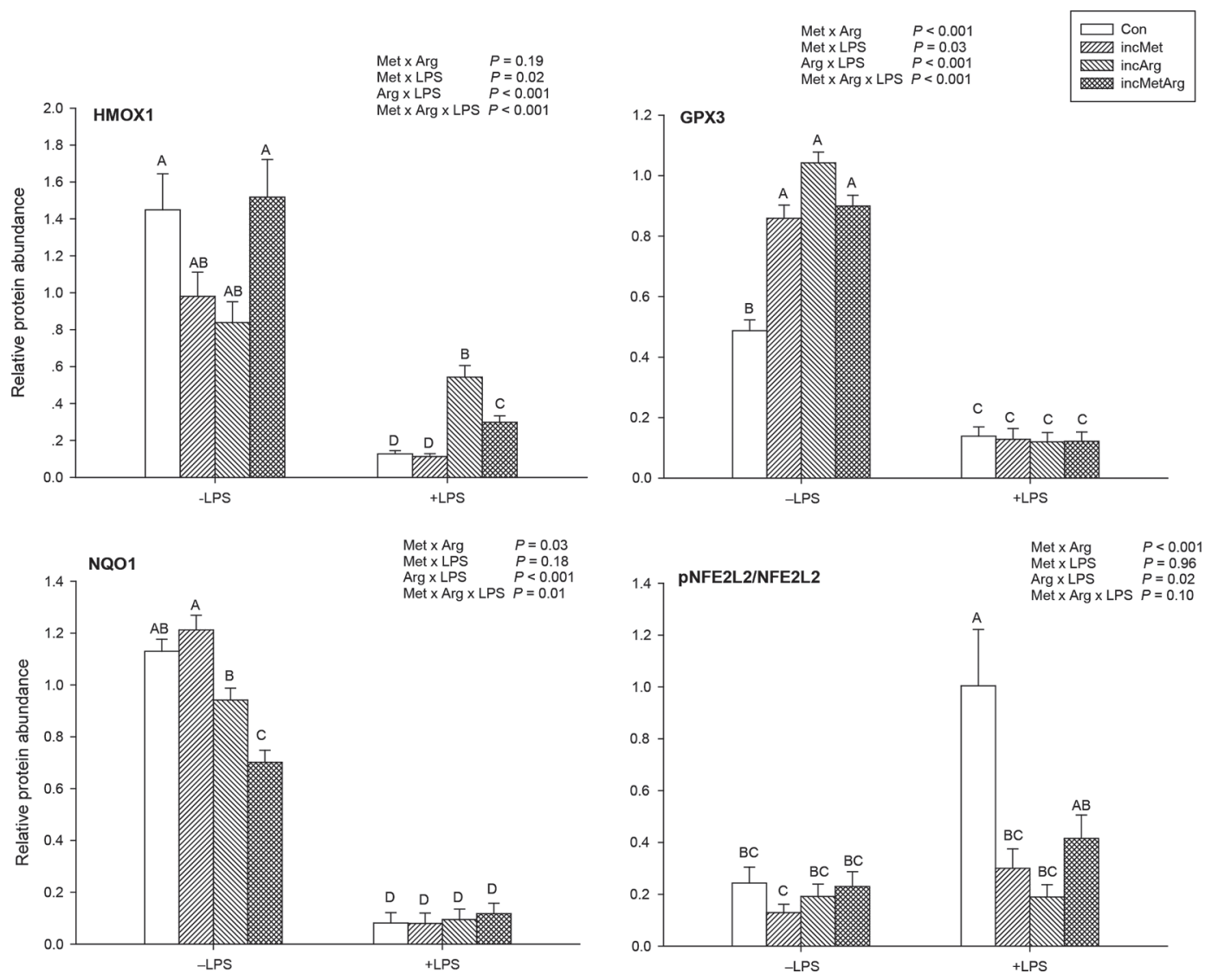

Figure 4. Effects of incubating primary bovine mammary cells with the ideal AA profile Lys:Met 2.9:1 and Lys:Arg 2:1 (Con) or with Lys: Met 2.5:1 and Lys:Arg 2:1 (incMet), Lys:Met 2.9:1 and Lys:Arg 1:1 (incArg), or Lys:Met 2.5:1 and Lys:Arg 1:1 (incMetArg) on abundance of proteins related to NFE2L2 signaling, in response to LPS challenge (+LPS = LPS challenge; - LPS $=$ no LPS stimulation). All data are reported as relative protein abundance of the target versus GAPDH, which served as the internal control. Different uppercase letters indicate significant differences between treatments $(P<0.05)$ with a significant triple interaction (Met $\times \operatorname{Arg} \times \operatorname{LPS} P<0.05)$, and error bars denote SEM. NFE2L2 = nuclear factor (erythroid-derived 2)-like 2; pNFE2L2 = phosphorylated NFE2L2; GPX3 = glutathione peroxidase 3; NQO1 = $\mathrm{NAD}(\mathrm{P}) \mathrm{H}$ dehydrogenase [quinone] 1; HMOX1, heme oxygenase (decycling) 1.

incArg during LPS stimulation suggests that Arg may modulate proinflammatory cytokine abundance triggered by LPS through intermediates in the ARG-ODC pathway. For instance, the upregulation of inducible NOS2 (a target of NF- $\kappa \mathrm{B}$ ), which catalyzes the generation of NO (Aldieri et al., 2003), was attenuated in cultures with incArg during LPS simulation. The polyamine spermine (produced during metabolism of Arg in the ARG-ODC pathway) can attenuate the release of proinflammatory cytokines (Zhang et al., 1997) and has also been observed to downregulate NOS2 in LPS-stimulated macrophages (Mössner et al., 2001). Although not measured in the present study, our previous work with immortalized mammary cells revealed alterations in polyamine concentrations in response to supply of EAA (Dong et al., 2018). Thus, it is possible that increased spermine production in the present study suppressed NF- $\kappa$ B activation and attenuated ex- pression of NOS2 and CXCL2. Further work is needed to elucidate the relationship between Arg, spermine, and their anti-inflammatory effects.

Toll-like receptor 2 and TLR4 are 2 important pattern-recognition receptors that play a crucial role in the innate immune system (Mukherjee et al., 2016). After binding ligands such as LPS, they initiate the activation of NF- $\kappa \mathrm{B}$ and other transcription factors, triggering the expression of cytokines such as IL-6 (Oeckinghaus et al., 2011). The fact that culture with incMet+LPS tended to further downregulate TLR \& from the results observed with Con+LPS is contrary to previous work with primary goat mammary epithelial cells or bovine mammary cells, in which LPS stimulation upregulated TLR4 expression (Bulgari et al., 2017; Wang et al., 2017a). The downregulation of TLR4 abundance in cultures with incMet during LPS stimulation would attenuate the signal transduction initiated by LPS, 
including that related to proinflammatory responses. When excessively activated, this type of response might be harmful for the mammary gland during infection. Thus, the downregulation of TLR 4 suggested a protective effect of increased Met supply during LPS challenge.

Methionine is a potential precursor for the biosynthesis of GSH through the transsulfuration pathway (Lu, 2013), and data have suggested that GSH not only is a major antioxidant but also participates in the regulation of cell proliferation, apoptosis, and immune function (Lu, 2013). Additionally, the systemic inflammatory response initiated by LPS in rats was significantly attenuated by increased supply of GSH (Sun et al., 2006). Thus, it is possible that downregulation of $I L 1 B$ and NOS2 in response to increased supply of Met during LPS challenge may have been associated with greater concentration of GSH, as detected previously in bovine hepatocytes challenged with LPS (Zhang and White, 2017). Interleukin $1 \beta$ is a proinflammatory cytokine, and its excessive expression is related to pathophysiological changes during inflammatory diseases (Ren and Torres, 2009). Nitric oxide is a product of NOS2 and plays an important role in homeostasis and normal function of organisms. However, overt production of NO leads to inflammation and tissue damage (Sharma et al., 2007). Thus, downregulation of $I L 1 B$ and NOS2, together with lower activity of NF- $\mathrm{KB}$ and TLR 4 expression in cultures with incMet+LPS, compared with Con+LPS, suggests a protective effect of Met. The anti-inflammatory effect of Met through its role in GSH synthesis has also been previously demonstrated in periparturient cows (Osorio et al., 2014), but further work is needed to elucidate the mechanisms involved.

The nuclear transcription factor NFE2L2 is a redoxsensitive transcription factor, and a cascade of events lead to increases in mRNA and protein abundance of several targets associated with the antioxidant response upon activation, including the reductase NQO1, GPX3, and SOD (Pias et al., 2003; Ruiz et al., 2013). Enzymes such as GPX3 are major scavengers of reactive oxygen species (An et al., 2018). Although the ratio of pNFE2L2 to total NFE2L2 protein was upregulated in response to LPS challenge, the increased activity of NFE2L2 may not have been enough to counteract the marked reduction in total NFE2L2 protein, resulting in an overall downregulation of NQO1, GPX3, and HMOX1 [another target of NFE2L2 (Prestera et al., 1995; Maines and Ewing, 1996)] protein abundance in response to LPS challenge. Similar findings were also reported by Shi et al. (2016), who found that LPS downregulated the activity of the key targets of NFE2L2 such as GPX and SOD.
Because a key function of NQO1 and GPX3 is to suppress the generation of reactive oxygen species (Ross and Siegel, 2018), and HMOX1 possesses both antiinflammatory (Piantadosi et al., 2011) and antioxidative properties, the LPS-challenged cells might have experienced a more pronounced state of oxidative stress. Such effect could not be alleviated by increased supply of Met or Arg. Thus, increased Met or Arg supply had little effect on the antioxidative response during LPS stimulation. The downregulation of the cell cycle regulator ATG7 confirms alterations in cellular function by the proinflammatory cascade induced by LPS.

\section{CONCLUSIONS}

Although the decrease in abundance and activity of NFE2L2 upon LPS stimulation of BMEC led to a dampening of the antioxidant response, increasing the supply of Met and Arg was effective in attenuating the proinflammatory response by preventing the activation of NF- $\kappa \mathrm{B}$ and associated proinflammatory cytokines. Transcriptional changes suggest that increased synthesis of polyamines and GSH by Arg and Met, respectively, helped attenuate those responses. Greater supply of Met and Arg did not enhance the antioxidant response during LPS challenge. Further work is needed to fully elucidate the mechanisms by which polyamines and GSH may attenuate inflammatory responses in BMEC.

\section{ACKNOWLEDGMENTS}

H. Dai and L. Hu received scholarships from China Scholarship Council (Beijing, China) to undertake PhD and MS training at the University of Illinois, Urbana.

\section{REFERENCES}

Akira, S. 2009. Pathogen recognition by innate immunity and its signaling. Proc. Jpn. Acad. Ser. B. Phys. Biol. Sci. 85:143-156.

Aldieri, E., D. Atragene, L. Bergandi, C. Riganti, C. Costamagna, A. Bosia, and D. Ghigo. 2003. Artemisinin inhibits inducible nitric oxide synthase and nuclear factor NF-kB activation. FEBS Lett. 552:141-144.

An, B. C., Y. D. Choi, I. J. Oh, J. H. Kim, J. I. Park, and S. W. Lee. 2018. GPx3-mediated redox signaling arrests the cell cycle and acts as a tumor suppressor in lung cancer cell lines. PLoS One 13:e0204170.

Bansal, V., and J. B. Ochoa. 2003. Arginine availability, arginase, and the immune response. Curr. Opin. Clin. Nutr. Metab. Care 6:223-228.

Batistel, F., J. M. Arroyo, C. I. M. Garces, E. Trevisi, C. Parys, M. A. Ballou, F. C. Cardoso, and J. J. Loor. 2018. Ethyl-cellulose rumen-protected methionine alleviates inflammation and oxidative stress and improves neutrophil function during the periparturient period and early lactation in Holstein dairy cows. J. Dairy Sci. 101:480-490.

Bröer, S., and M. Palacin. 2011. The role of amino acid transporters in inherited and acquired diseases. Biochem. J. 436:193-211. 
Bronte, V., and P. Zanovello. 2005. Regulation of immune responses by L-arginine metabolism. Nat. Rev. Immunol. 5:641-654.

Brosnan, J. T., and M. E. Brosnan. 2006. The sulfur-containing amino acids: An overview. J. Nutr. 136(Suppl.):1636S-1640S.

Bulgari, O., X. Dong, A. L. Roca, A. M. Caroli, and J. J. Loor. 2017. Innate immune responses induced by lipopolysaccharide and lipoteichoic acid in primary goat mammary epithelial cells. J. Anim. Sci. Biotechnol. 8:29.

Closs, E. I., J.-S. Scheld, M. Sharafi, and U. Förstermann. 2000. Substrate supply for nitric-oxide synthase in macrophages and endothelial cells: Role of cationic amino acid transporters. Mol. Pharmacol. 57:68-74.

Dasgupta, T., R. P. Hebbel, and D. K. Kaul. 2006. Protective effect of arginine on oxidative stress in transgenic sickle mouse models. Free Radic. Biol. Med. 41:1771-1780.

de Andrade Bernal Fagiani, M., A. Fluminhan, F. de Azevedo Mello, D. Yabuki, G. V. Goncalves, L. K. Tsujigushi, L. G. Pereria, K. A. da Silva, S. B. B. da Silva, C. L. Santarem, P. F. I. Giozo, and L. S. L. de Souza Reis. 2019. L-Arginine minimizes immunosuppression and prothrombin time and enhances the genotoxicity of 5-fluorouracil in rats. Nutrition 66:94-100. https://doi.org/10.1016/j.nut 2019.04.012

Dong, X., Z. Zhou, B. Saremi, A. Helmbrecht, Z. Wang, and J. J. Loor. 2018. Varying the ratio of Lys:Met while maintaining the ratios of Thr:Phe, Lys:Thr, Lys:His, and Lys:Val alters mammary cellular metabolites, mammalian target of rapamycin signaling, and gene transcription. J. Dairy Sci. 101:1708-1718.

Elazar, S., E. Gonen, A. Livneh-Kol, I. Rosenshine, and N. Y. Shpigel. 2010. Essential role of neutrophils but not mammary alveolar macrophages in a murine model of acute Escherichia coli mastitis. Vet. Res. 41:53.

Haque, M. N., H. Rulquin, A. Andrade, P. Faverdin, J. L. Peyraud, and S. Lemosquet. 2012. Milk protein synthesis in response to the provision of an "ideal" amino acid profile at 2 levels of metabolizable protein supply in dairy cows. J. Dairy Sci. 95:5876-5887.

Haque, M. N., H. Rulquin, and S. Lemosquet. 2013. Milk protein responses in dairy cows to changes in postruminal supplies of arginine, isoleucine, and valine. J. Dairy Sci. 96:420-430. https://doi .org/10.3168/jds.2012-5610.

He, X., W. Liu, M. Shi, Z. Yang, X. Zhang, and P. Gong. 2017. Docosahexaenoic acid attenuates LPS-stimulated inflammatory response by regulating the PPAR $\gamma / \mathrm{NF}-\mathrm{\kappa B}$ pathways in primary bovine mammary epithelial cells. Res. Vet. Sci. 112:7-12.

Hosseini, A., M. R. Tariq, F. Trindade da Rosa, J. Kesser, Z. Iqbal, O. Mora, H. Sauerwein, J. K. Drackley, E. Trevisi, and J. J. Loor. 2015. Insulin sensitivity in adipose and skeletal muscle tissue of dairy cows in response to dietary energy level and 2,4-thiazolidinedione (TZD). PLoS One 10:e142633.

Jensen, A., M. Figueiredo-Larsen, R. Holm, M. L. Broberg, B. Brodin, and C. U. Nielsen. 2014. PAT1 (SLC36A1) shows nuclear localization and affects growth of smooth muscle cells from rats. Am. J. Physiol. Endocrinol. Metab. 306:E65-E74. https://doi.org/10 .1152/ajpendo.00322.2013.

Lean, I. J., M. B. de Ondarza, C. J. Sniffen, J. E. P. Santos, and K. E. Griswold. 2018. Meta-analysis to predict the effects of metabolizable amino acids on dairy cattle performance. J. Dairy Sci. 101:340-364

Li, P., Y. L. Yin, D. Li, S. W. Kim, and G. Wu. 2007. Amino acids and immune function. Br. J. Nutr. 98:237-252.

Li, S., A. Hosseini, M. Danes, C. Jacometo, J. Liu, and J. J. Loor. 2016. Essential amino acid ratios and mTOR affect lipogenic gene networks and miRNA expression in bovine mammary epithelial cells. J. Anim. Sci. Biotechnol. 7:44.

Li, X., W. Huang, J. Gu, X. Du, L. Lei, X. Yuan, G. Sun, Z. Wang, X. Li, and G. Liu. 2015. SREBP-1c overactivates ROS-mediated hepatic NF- $\mathrm{B}$ inflammatory pathway in dairy cows with fatty liver. Cell. Signal. 27:2099-2109.

Lu, S. C. 2013. Glutathione synthesis. Biochim. Biophys. Acta 1830:3143-3153.

Maines, M. D., and J. F. Ewing. 1996. Stress response of the rat testis: In situ hybridization and immunohistochemical analysis of heme oxygenase-1 (HSP32) induction by hyperthermia. Biol. Reprod. 54:1070-1079.

Mössner, J., R. Hammermann, and K. Racke. 2001. Concomitant down-regulation of L-arginine transport and nitric oxide (NO) synthesis in rat alveolar macrophages by the polyamine spermine. Pulm. Pharmacol. Ther. 14:297-305.

Moyes, K. M., J. K. Drackley, D. E. Morin, and J. J. Loor. 2010. Greater expression of TLR2, TLR4, and IL6 due to negative energy balance is associated with lower expression of HLA-DRA and HLA-A in bovine blood neutrophils after intramammary mastitis challenge with Streptococcus uberis. Funct. Integr. Genomics 10:53-61. https://doi.org/10.1007/s10142-009-0154-7.

Mukherjee, S., S. Karmakar, and S. P. Babu. 2016. TLR2 and TLR4 mediated host immune responses in major infectious diseases: A review. Braz. J. Infect. Dis. 20:193-204.

NRC (National Research Council). 2001. Nutrient Requirements of Dairy Cattle. 7th rev. ed. Natl. Acad. Press, Washington, DC.

Oeckinghaus, A., M. S. Hayden, and S. Ghosh. 2011. Crosstalk in NF$\mathrm{\kappa B}$ signaling pathways. Nat. Immunol. 12:695.

Oliver, S. P., and T. Bushe. 1987. Growth inhibition of Escherichia coli and Klebsiella pneumoniae during involution of the bovine mammary gland: Relation to secretion composition. Am. J. Vet. Res. 48:1669-1673.

Osorio, J. S., E. Trevisi, P. Ji, J. K. Drackley, D. Luchini, G. Bertoni, and J. J. Loor. 2014. Biomarkers of inflammation, metabolism, and oxidative stress in blood, liver, and milk reveal a better immunometabolic status in peripartal cows supplemented with Smartamine M or MetaSmart. J. Dairy Sci. 97:7437-7450.

Pegg, A. E., H. Xiong, D. J. Feith, and L. M. Shantz. 1998. S-adenosylmethionine decarboxylase: Structure, function and regulation by polyamines. Biochem. Soc. Trans. 26:580-586.

Piantadosi, C. A., C. M. Withers, R. R. Bartz, N. C. MacGarvey, P. Fu, T. E. Sweeney, K. E. Welty-Wolf, and H. B. Suliman. 2011. Heme oxygenase-1 couples activation of mitochondrial biogenesis to anti-inflammatory cytokine expression. J. Biol. Chem. 286:16374-16385.

Pias, E. K., O. Y. Ekshyyan, C. A. Rhoads, J. Fuseler, L. Harrison, and T. Y. Aw. 2003. Differential effects of superoxide dismutase isoform expression on hydroperoxide-induced apoptosis in PC-12 cells. J. Biol. Chem. 278:13294-13301.

Popovic, P. J., H. J. Zeh III, and J. B. Ochoa. 2007. Arginine and immunity. J. Nutr. 137(Suppl 2):1681S-1686S.

Prestera, T., P. Talalay, J. Alam, Y. I. Ahn, P. J. Lee, and A. M. K. Choi. 1995. Parallel induction of heme oxygenase-1 and chemoprotective phase 2 enzymes by electrophiles and antioxidants: Regulation by upstream antioxidant-responsive elements (ARE). Mol. Med. 1:827-837.

Ren, K., and R. Torres. 2009. Role of interleukin-1 $\beta$ during pain and inflammation. Brain Res. Rev. 60:57-64.

Ross, D., and D. Siegel. 2018. NQO1 in protection against oxidative stress. Curr. Opin. Toxicol. 7:67-72.

Ruiz, S., P. E. Pergola, R. A. Zager, and N. D. Vaziri. 2013. Targeting the transcription factor Nrf2 to ameliorate oxidative stress and inflammation in chronic kidney disease. Kidney Int. 83:1029-1041.

Salama, A. A. K., M. Duque, L. Wang, K. Shahzad, M. Olivera, and J. J. Loor. 2019. Enhanced supply of methionine or arginine alters mechanistic target of rapamycin signaling proteins, messenger RNA, and microRNA abundance in heat-stressed bovine mammary epithelial cells in vitro. J. Dairy Sci. 102:2469-2480.

Sharma, J. N., A. Al-Omran, and S. S. Parvathy. 2007. Role of nitric oxide in inflammatory diseases. Inflammopharmacology 15:252259.

Shi, H., Y. Guo, Y. Liu, B. Shi, X. Guo, L. Jin, and S. Yan. 2016. The in vitro effect of lipopolysaccharide on proliferation, inflammatory factors and antioxidant enzyme activity in bovine mammary epithelial cells. Anim Nutr 2:99-104.

Sun, S., H. Zhang, B. Xue, Y. Wu, J. Wang, Z. Yin, and L. Luo. 2006. Protective effect of glutathione against lipopolysaccharide-induced inflammation and mortality in rats. Inflamm. Res. 55:504-510. https://doi.org/10.1007/s00011-006-6037-7. 
Suzuki, T., and M. Yamamoto. 2015. Molecular basis of the Keap1Nrf2 system. Free Radic. Biol. Med. 88:93-100.

Vailati-Riboni, M., T. Xu, B. Qadir, R. Bucktrout, C. Parys, and J. J. Loor. 2019. In vitro methionine supplementation during lipopolysaccharide stimulation modulates immunometabolic gene network expression in isolated polymorphonuclear cells from lactating Holstein cows. J. Dairy Sci. 102: 8343-8351. https://doi.org/10.3168/ jds.2018-15737.

Wang, M., B. Xu, H. Wang, D. Bu, J. Wang, and J.-J. Loor. 2014. Effects of arginine concentration on the in vitro expression of casein and mTOR pathway related genes in mammary epithelial cells from dairy cattle. PLoS One 9:e95985.

Wang, W., X. Hu, P. Shen, N. Zhang, and Y. Fu. 2017a. Sodium houttuyfonate inhibits LPS-induced inflammatory response via suppressing TLR4/NF-kB signaling pathway in bovine mammary epithelial cells. Microb. Pathog. 107:12-16.

Wang, Y., X. Zhang, Z. Wei, J. Wang, Y. Zhang, M. Shi, Z. Yang, and Y. Fu. 2017b. Platycodin D suppressed LPS-induced inflammatory response by activating LXRalpha in LPS-stimulated primary bovine mammary epithelial cells. Eur. J. Pharmacol. 814:138-143.

Wu, G. 2013. Functional amino acids in nutrition and health. Amino Acids 45:407-411.

Wu, T., C. Wang, L. Ding, Y. Shen, H. Cui, M. Wang, and H. Wang. 2016. Arginine relieves the inflammatory response and enhances the casein expression in bovine mammary epithelial cells induced by lipopolysaccharide. Mediators Inflamm. 2016:9618795.

Zadoks, R. N., J. R. Middleton, S. McDougall, J. Katholm, and Y. H. Schukken. 2011. Molecular epidemiology of mastitis pathogens of dairy cattle and comparative relevance to humans. J. Mammary Gland Biol. Neoplasia 16:357-372.

Zhang, M., T. Caragine, H. Wang, P. S. Cohen, G. Botchkina, K. Soda, M. Bianchi, P. Ulrich, A. Cerami, B. Sherry, and K. J. Tracey. 1997. Spermine inhibits proinflammatory cytokine synthesis in human mononuclear cells: A counterregulatory mechanism that restrains the immune response. J. Exp. Med. 185:1759-1768.

Zhang, Q., and H. M. White. 2017. Regulation of inflammation, antioxidant production, and methyl-carbon metabolism during methionine supplementation in lipopolysaccharide-challenged neonatal bovine hepatocytes. J. Dairy Sci. 100:8565-8577.

Zhao, F. F., T. Y. Wu, H. R. Wang, L. Y. Ding, G. Ahmed, H. W. Li, W. Tian, and Y. Z. Shen. 2018. Jugular arginine infusion relieves lipopolysaccharide-triggered inflammatory stress and improves immunity status of lactating dairy cows. J. Dairy Sci. 101:5961-5970.

Zhou, Z., O. Bulgari, M. Vailati-Riboni, E. Trevisi, M. A. Ballou, F. C. Cardoso, D. N. Luchini, and J. J. Loor. 2016. Rumen-protected methionine compared with rumen-protected choline improves immunometabolic status in dairy cows during the peripartal period. J. Dairy Sci. 99:8956-8969.

\section{ORCIDS}

D. N. Coleman @ https://orcid.org/0000-0002-8783-095X

X. Shen (1) https://orcid.org/0000-0002-3020-5010

J. J. Loor ๑ https://orcid.org/0000-0003-1586-4365 